\title{
Slow Passage through Multiple Parametric Resonance Tongues
}

\author{
JACQUELINE BRIDGE \\ Department of Mechanical Engineering, University of The West Indies, St. Augustine, Trinidad
}

RICHARD RAND

Department Theoretical and Applied Mechanics, Cornell University, Ithaca, NY 14853, USA

(rhr2@cornell.edu)

SI MOHAMED SAH

Faculty of Sciences, Ä̈n Chock, BP 5366 Maârif, Casablanca, Morocco

(Received 28 March 2008; accepted 22 July 2008)

\begin{abstract}
This work concerns linear parametrically excited systems that involve multiple resonances. The property of such systems is that if the parameters are fixed and lie inside a resonance tongue, the motion becomes unbounded as time goes to infinity. In this work we consider what happens when the parameters are not fixed, but rather are constrained to vary slowly in time, passing into and out of the resonance tongues. One might expect that during the time in which the motion lies inside a tongue the solution grows, and that the slower the passage through the tongue the more time is spent inside the tongue, and the larger the resulting growth. We show that this is not always the case. In particular we investigate the effect of initial conditions and relative forcing amplitudes on the growth or amplification of the solution. We address the problem of how to choose these parameters so as to minimize growth (i.e., to de-amplify the solution) after passage through multiple tongues.
\end{abstract}

Keywords: Parametric excitation, slow passage, multiple resonances, amplification.

\section{INTRODUCTION}

Parametrically excited dynamical mathematical models are widely used to represent physical and engineering systems, particularly the response of mechanical, elastic and hydrodynamic systems to time-varying loads. If the variation is sinusoidal, then the system can generally be reduced to the Mathieu equation, and is said to involve a single resonance. However, if the variation has more than one frequency component, each of which is incommensurate with the others, then the system is said to involve multiple resonances. The simplest example of such a system is the Mathieu equation with two forcing frequencies, 


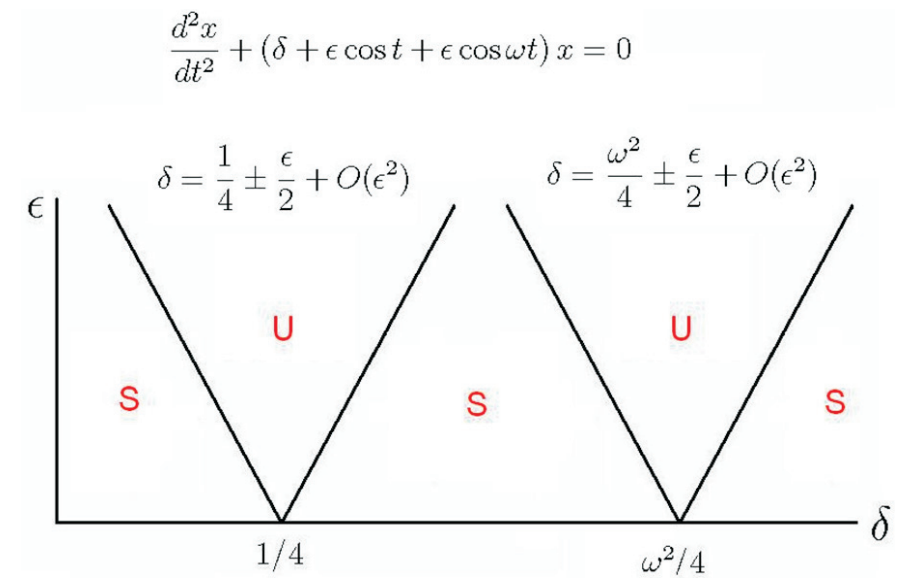

Figure 1. Form of the two large resonant tongues in a Mathieu equation with two forcing frequencies.

$$
\frac{d^{2} x}{d t^{2}}+\left(\delta+\epsilon\left(\alpha_{1} \cos \omega_{1} t+\alpha_{2} \cos \omega_{2} t\right)\right) x=0 .
$$

In this paper, we consider the effect of a slow linear variation of the parameter $\delta$, as may be expected to occur during slow start-up or shut-down of engineering or physical systems. In the case that $\delta$ is a constant and $\epsilon$ is a small parameter, equation (1) exhibits two large resonance tongues in the $\delta-\epsilon$ plane emerging from the $\delta$ axis at $\delta=\omega_{1}^{2} / 4$ and $\delta=\omega_{2}^{2} / 4$, respectively (Zounes and Rand, 1998). In addition there are an infinitude of smaller resonance tongues. We will concentrate on the effect of passage through the primary resonances only. Although there may be additional effects associated with moving through the higher-order tongues, numerical experiments have shown that these will involve minimal amplification. Consequently, we will not consider their effect in this paper. We also limit our analysis to cases in which $\omega_{1}$ is sufficiently smaller than $\omega_{2}$, resulting in well-separated tongues. In equation (1), $\alpha_{1}$ and $\alpha_{2}$ are parameters which determine the relative amplitudes of the forcing functions. See Figure 1 where the tongues for the case $\alpha_{1}=\alpha_{2}=\omega_{1}=1, \omega_{2}=\omega$ are displayed.

In the case where $\delta$ is a constant, points lying inside the resonant tongues will correspond to systems which have solutions $x(t)$ which grow exponentially in time $t$ and are often referred to as being unstable. If, on the other hand, $\delta$ varies with $t$, it may pass through one or more "resonance tongues", during which time the solution $x(t)$ may be expected to grow. (There are no real resonance tongues in the slow flow equation; the term is used to characterize the two-frequency excitation in equation (1) in the language of the regular Mathieu equation.) It may be expected that the slower the passage through a resonance tongue, or the wider the tongue, the more time is spent inside the unstable region, and the larger is the resulting growth. This question has been addressed in a previous work ( $\mathrm{Ng}$ et al., 2003) which dealt with slow passage through a single tongue. We briefly review the results in $\mathrm{Ng}$ et al. (2003), where the following equation was studied: 


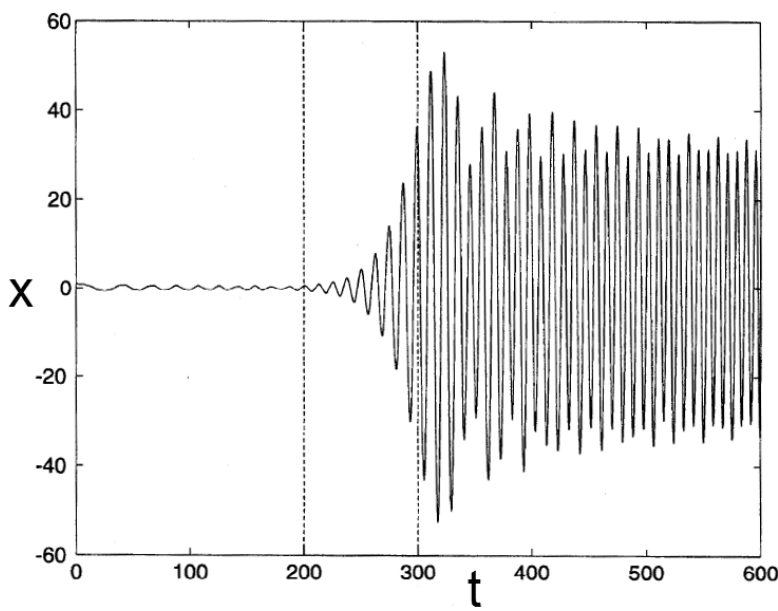

Figure 2. Numerical integration of equation (2) for $\sigma=0, \epsilon=0.1, \alpha=1, \mu=0.1, x(0)=1$ and $d x / d t(0)=0$. The dashed vertical lines show nominal times at which the motion enters and exits the resonant tongue ( $\mathrm{Ng}$ et al., 2003).

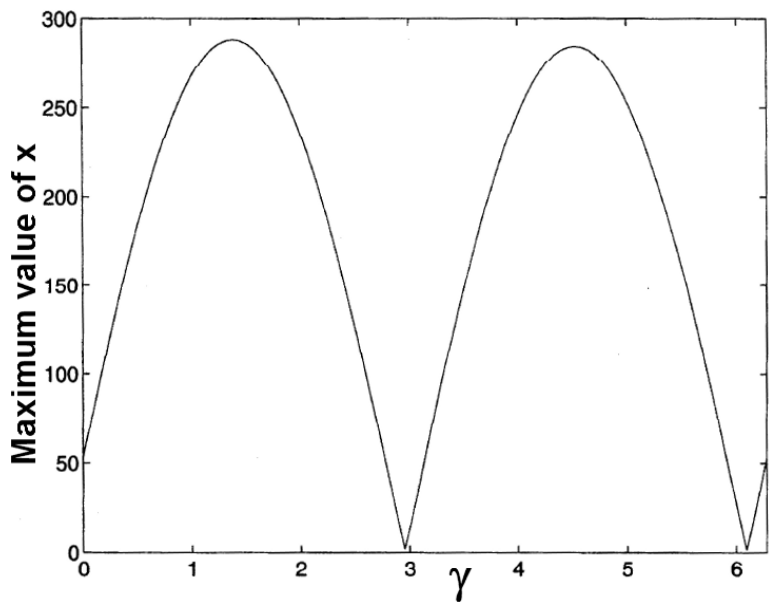

Figure 3. Maximum value of $x$ as a function of initial-condition phase $\gamma$ for equation (2) (Ng et al., 2003). Results obtained by numerically integrating equation (2) for $\sigma=0, \epsilon=0.1, \alpha=1, \mu=0.1, x(0)=\cos \gamma$ and $d x / d t(0)=\sin \gamma$.

$$
\frac{d^{2} x}{d t^{2}}+(\delta+\epsilon \alpha \cos t) x=0 \quad \text { where } \quad \delta=\sigma+\epsilon^{2} \mu t
$$

Figure 2 shows the results of numerical integration of equation (2) in which $\sigma=0, \epsilon=0.1$, $\alpha=1$ and $\mu=0.1$ for the initial conditions $x(0)=1$ and $d x / d t(0)=0$. Figure 3 shows the effect of changing initial conditions by varying $\gamma$ in the equations 


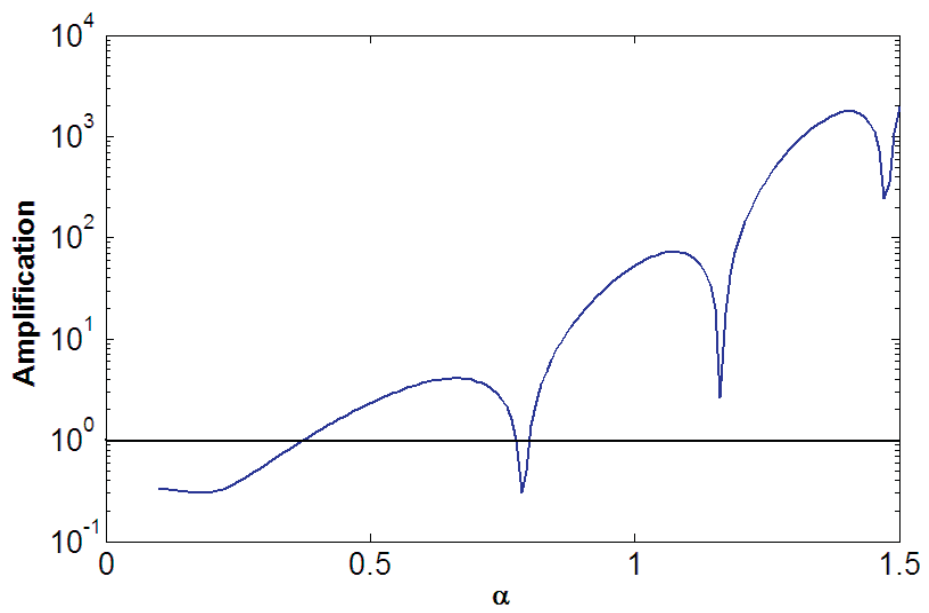

Figure 4. Maximum value of $x$ as a function of the coefficient of the parametric excitation, $\alpha$. Results obtained by numerically integrating equation (2) for $\sigma=0, \epsilon=0.1, \mu=0.1, x(0)=1$ and $d x / d t(0)=0$. Points above the horizontal line at $10^{0}=1$ represent amplification, while points below the line represent de-amplification.

$$
x(0)=\cos \gamma \quad \text { and } \quad \frac{d x}{d t}(0)=\sin \gamma .
$$

From Figure 3 we see that the phase $\gamma$ of the initial conditions has an important influence on the overall growth involved in passage through a single resonance tongue. $\mathrm{In} \mathrm{Ng}$ et al. (2003), this growth effect has been called amplification. Note that while most initialcondition phases $\gamma$ lead to an increase in amplitude, there is a small range of $\gamma$ values close to $\gamma=2.9558$ which lead to de-amplification.

Finally we note from Figure 4, that the amplification is dependent on the magnitude of the parametric excitation, $\alpha$. The graph is not monotonically increasing; there are a series of local maxima and minima. The reason for this phenomenon, which was not examined in $\mathrm{Ng}$ et al. (2003), will be discussed later on in this paper.

\section{TWO TONGUES}

We first consider slow passage through a two-tongue system in the form of equation (1) with $\alpha_{1}=\alpha_{2}=1, \omega_{1}=2$ and $\omega_{2}=2 \sqrt{2}$ :

$$
\frac{d^{2} x}{d t^{2}}+(\delta+\epsilon(\cos 2 t+\cos 2 \sqrt{2} t)) x=0 \quad \text { where } \quad \delta=\sigma+\epsilon^{2} \mu t .
$$

Here $\omega_{1}$ and $\omega_{2}$ have been chosen so that the associated tongues are centered at $\delta=1$ and $\delta=2$, respectively. 


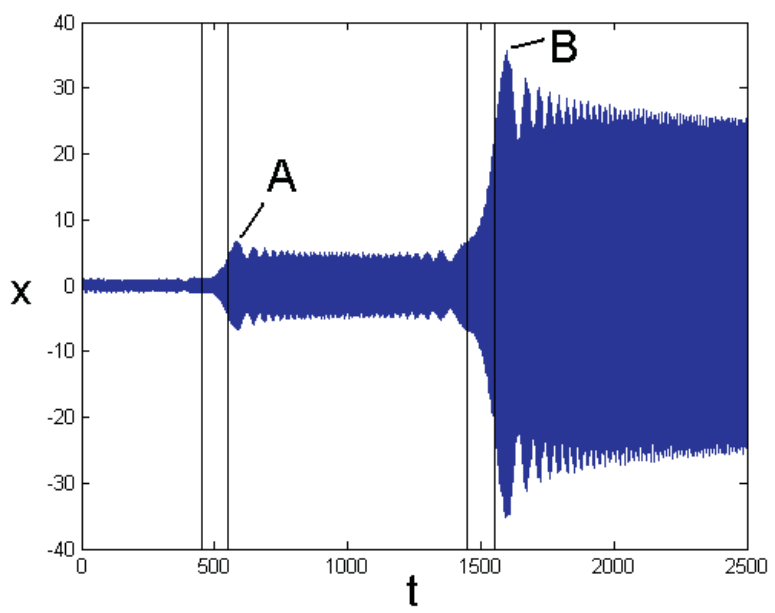

Figure 5. Numerical integration of equation (4) for $\sigma=0.5, \epsilon=0.1, \mu=0.1$ and for the initial conditions $x(0)=1$ and $d x / d t(0)=0$. The vertical lines show nominal times at which the motion enters and exits the two resonant tongues. The letters $\mathrm{A}$ and $\mathrm{B}$ refer to the local maximum values of $x$ associated respectively with the first and second resonant tongues, cf. Figure 6.

Figure 5 shows the results of numerical integration of equation (4) in which $\sigma=0.5$, $\epsilon=0.1$ and $\mu=0.1$ for the initial conditions $x(0)=1$ and $d x / d t(0)=0$. We may say that Figure 5 is the two-tongue version of Figure 2. As the motion passes through each of the tongues, local amplification is observed, and the final amplification is the product of the two individual amplifications.

Let us next consider the two-tongue version of Figure 3, that is a plot of the maximum values of $x$ as a function of initial-condition phase $\gamma$. This is displayed in Figure 6 where the curve marked A refers to the local maximum achieved after passage through the first resonant tongue, and where the curve marked $\mathrm{B}$ refers to the local maximum achieved after passage through the second resonant tongue. Here the curve marked B/A shows the ratio of these maximum values and thus represents the amplification which occurs in the second tongue. Note that although the amplification associated with passage through the first resonant tongue is highly dependent on the initial condition phase $\gamma$ both here and in the case of the single tongue system, Figure 3, the amplification which occurs in the second tongue is not dependent on $\gamma$, except in the narrow interval where both $A$ and $B$ are smallest.

We also consider the effect of changing the values of the forcing amplitudes $\alpha_{1}$ and $\alpha_{2}$ in equation (1). We use the following generalization of equation (4) as an example:

$$
\frac{d^{2} x}{d t^{2}}+\left(\delta+\epsilon\left(\alpha_{1} \cos 2 t+\alpha_{2} \cos 2 \sqrt{2} t\right)\right) x=0 \quad \text { where } \quad \delta=\sigma+\epsilon^{2} \mu t
$$

Here we may think of each $\alpha_{i}$ as changing the effective value of $\epsilon$ to $\epsilon_{i}=\epsilon \alpha_{i}$. Since $\epsilon$ controls the width of the resonance tongue, cf. Figure 1, the role of each $\alpha_{i}$ is to vary the width of the respective tongue. In the Introduction it was conjectured that the more time spent in the unstable region (resonance tongue), the larger would be the resulting growth. Conse- 




Figure 6. Maximum values of $x$ as a function of initial condition phase $\gamma$ for equation (4) with $\sigma=0.5$, $\epsilon=0.1$ and $\mu=0.1$. The curves marked $\mathrm{A}$ and $\mathrm{B}$ refer to the local maximum values of $x$ associated respectively with the first and second resonant tongues, cf. Figure 5. The curve marked B/A shows the ratio of these maximum values and thus represents the amplification which occurs in the second tongue. Results obtained by numerically integrating equation (4).

quently, our initial expectation is that as $\alpha_{i}$ is increased the amplification will also increase. To determine the validity of this expectation, equation (5) was numerically integrated. The parameters were set $(\sigma=0.5, \epsilon=0.1, \mu=0.1)$ and the initial conditions were chosen to be $x(0)=1$ and $d x / d t(0)=0$. Two separate runs were performed: (a) $\alpha_{1}$ was held fixed at 1 while $\alpha_{2}$ was allowed to vary from 0.1 to 1.5 ; (b) $\alpha_{2}$ was held fixed at 1 while $\alpha_{1}$ was allowed to vary from 0.1 to 1.5 .

If the rate of growth of the solution was dependent only on the time spent in the resonance tongue, we would expect that as an $\alpha_{i}$ value is increased, there would be a monotonic increase in the amplification for fixed initial conditions. However, Figures 7 and 8 , which show the maximum values of $x$ as a function of the respective $\alpha_{i}$, tell a different story. For small values of each $\alpha_{i}$, we notice that the amplification is approximately equal to 1 , i.e. the resonance tongue has minimal effect on the amplitude of the motion. One can posit that this is so because the width of the tongue is very small and, consequently, the motion does not have enough time for substantial growth as it passes through. However in Figure 7, there is a curious occurrence at $\alpha_{2} \approx 0.65$; for this value the amplification is a local minimum in the curves marked $\mathrm{B}$ and $\mathrm{B} / \mathrm{A}$, and the response emerges from the second resonance tongue with a smaller amplitude than it entered with. Also notice that there is a local maximum for the amplification in the second tongue at $\alpha_{2} \approx 1.35$. Figure 8 exhibits three local maxima and two local minima for curve B, representing the overall amplification of the solution. One of the local minima occurs when there is a local minimum during passage through the first resonance tongue at $\alpha_{1} \approx 0.88$ and the other occurs when there is a local minimum during passage through the second resonance tongue at $\alpha_{1} \approx 1.19$.

Based on these numerical integrations, we see that the amplification depends on both the initial conditions and the respective coefficients of the parametric excitations, $\alpha_{i}$. The 


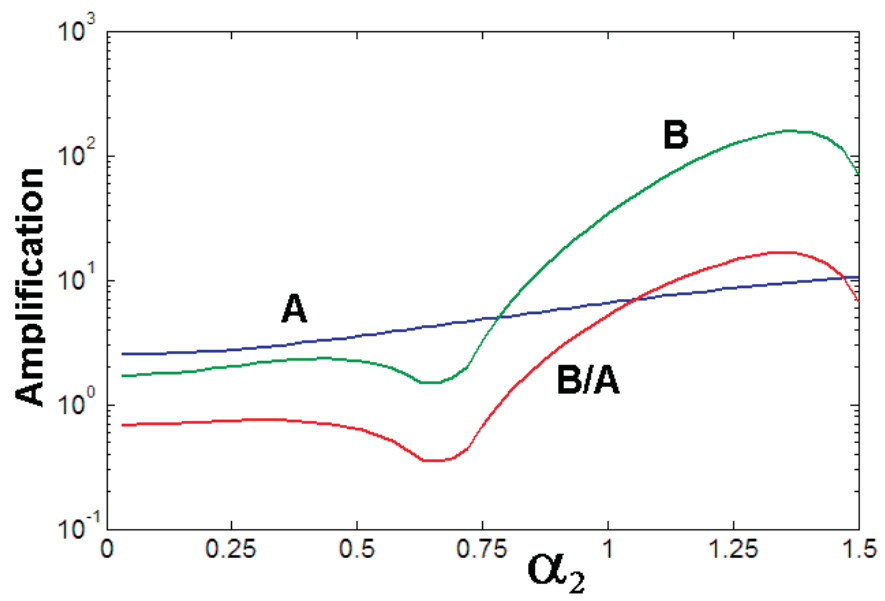

Figure 7. Maximum value of $x$ as a function of the coefficient of the parametric excitation in the second resonance tongue $\alpha_{2}$. The initial condition is chosen as $x(0)=1$ and $d x / d t(0)=0$. The curves marked $\mathrm{A}$ and $\mathrm{B}$ refer to the local maximum values of $x$ associated respectively with the first and second resonant tongues. The curve marked $\mathrm{B} / \mathrm{A}$ shows the ratio of these maximum values and thus represents the amplification which occurs in the second tongue. Results obtained by numerically integrating equation (5) for $\sigma=0.5, \epsilon=0.1, \mu=0.1, \alpha_{1}=1$.

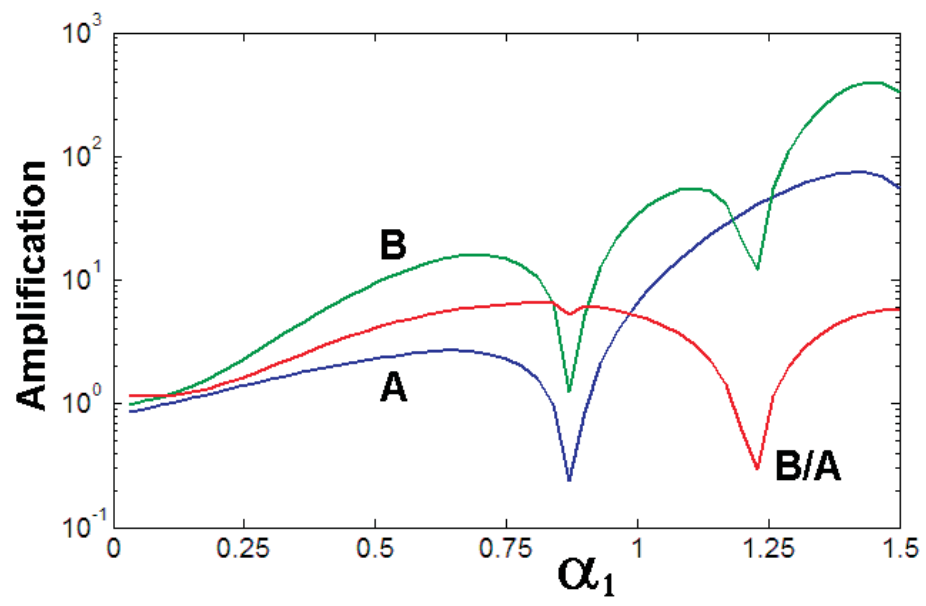

Figure 8. Maximum value of $x$ as a function of the coefficient of the parametric excitation in the first resonance tongue $\alpha_{1}$. The initial condition is chosen as $x(0)=1$ and $d x / d t(0)=0$. The curves marked $\mathrm{A}$ and $\mathrm{B}$ refer to the local maximum values of $x$ associated respectively with the first and second resonant tongues. The curve marked $\mathrm{B} / \mathrm{A}$ shows the ratio of these maximum values and thus represents the amplification which occurs in the second tongue. Results obtained by numerically integrating equation (5) for $\sigma=0.5, \epsilon=0.1, \mu=0.1, \alpha_{2}=1$. 


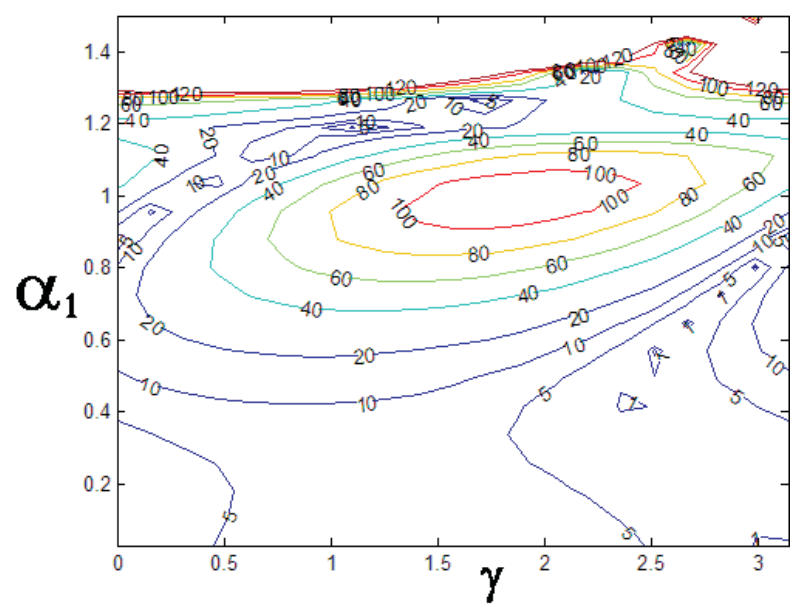

Figure 9. Contour plot of the maximum value of $x$ (i.e., point $\mathrm{B}$ in Figure 5) as a function of both initial condition phase $\gamma$ and the coefficient of the parametric excitation in the first resonance tongue $\alpha_{1}$. Results obtained by numerically integrating equation (5) for $\sigma=0.5, \epsilon=0.1, \mu=0.1, \alpha_{2}=1$.

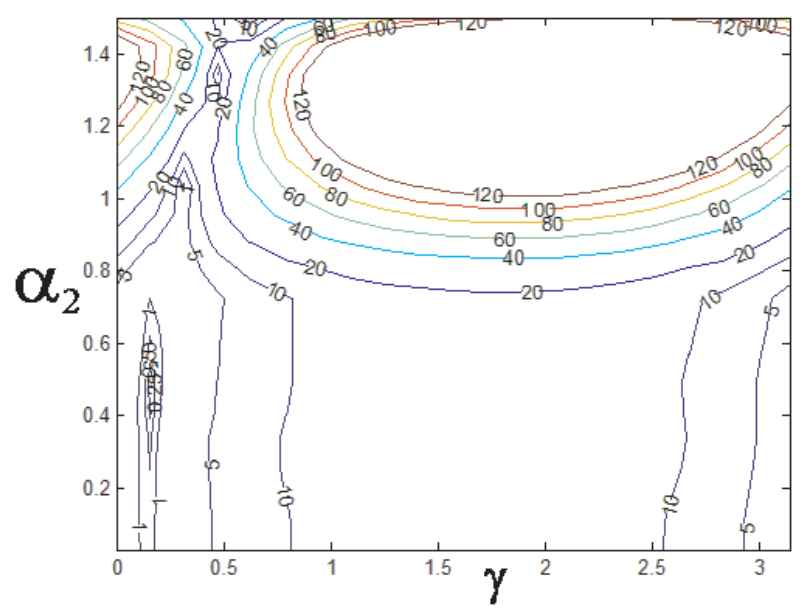

Figure 10. Contour plot of the maximum value of $x$ (i.e., point $\mathrm{B}$ in Figure 5) as a function of both initial condition phase $\gamma$ and the coefficient of the parametric excitation in the second resonance tongue $\alpha_{2}$. Results obtained by numerically integrating equation (5) for $\sigma=0.5, \epsilon=0.1, \mu=0.1, \alpha_{1}=1$.

contour plots in Figures 9 and 10 give an indication of how the system depends on these parameters. The line of minimum amplification does not coincide with the $\alpha_{i}=0$ line as would be expected if the amplification of the system depended only on the width of the resonance tongue. The Mathieu equation with two tongues, equation (1), is therefore seen to exhibit some interesting behavior. However, what is the underlying structure which causes this? In order to answer this question, we investigate the following simplified system. 


\section{SIMPLIFIED SYSTEM}

Our understanding of what is happening in equation (1) may be described in words as follows. In the neighborhood of each of the resonance tongues (i.e., when $\delta \approx \omega_{i}^{2} / 4$ for $i=1,2$ ), the respective cosine term $\epsilon \alpha_{i} \cos \omega_{i} t$ becomes important. Away from the resonant tongues, the cosine terms have little effect. Thus we are led to a simplified model which omits the cosine terms except in the neighborhood of their respective resonance. Furthermore, in the neighborhood of each one of the resonances, the model takes $\delta$ equal to its resonant value and omits the nonresonant cosine term. In equation (1), we may define the boundaries of the neighborhood of the resonance tongues to be $\delta=\omega_{i}^{2} / 4 \pm \epsilon \alpha_{i} / 2$, cf. Figure 1 . Thus in the case of equation (4), with $\sigma=0.5, \epsilon=0.1$ and $\mu=0.1$, our simplified system would take the form (cf. Figure 5)

$$
\begin{aligned}
x^{\prime \prime}+(0.5+0.001 t) x & =0 \text { for } 0<t<450, \\
x^{\prime \prime}+(1+0.1 \cos 2 t) x & =0 \text { for } 450<t<550, \\
x^{\prime \prime}+(0.5+0.001 t) x & =0 \text { for } 550<t<1450, \\
x^{\prime \prime}+(2+0.1 \cos 2 \sqrt{2} t) x & =0 \text { for } 1450<t<1550, \\
x^{\prime \prime}+(0.5+0.001 t) x & =0 \text { for } 1550<t .
\end{aligned}
$$

In order to glean as much understanding as possible out of this simplified model, we shall obtain solutions to equations (6)-(10) by using analytical techniques rather than numerical integration.

In the case of equations (6), (8) and (10), we may obtain an exact solution in terms of Airy functions. Airy's equation (Bender and Orszag, 1978) is

$$
\frac{d^{2} x}{d \tau^{2}}-\tau x=0
$$

and has the general solution

$$
x(\tau)=c_{1} \operatorname{Ai}(\tau)+c_{2} \operatorname{Bi}(\tau)
$$

where $c_{1}$ and $c_{2}$ are arbitrary constants.

Comparison of equations (11) and (6) shows that if we set $\tau=-50-0.1 t$ we obtain the general solution to equations (6),(8) and (10) in the form

$$
x(t)=c_{1} \operatorname{Ai}(-50-0.1 t)+c_{2} \operatorname{Bi}(-50-0.1 t) .
$$

Turning now to equations (7) and (9), we write these Mathieu equations in the form

$$
\frac{d^{2} x}{d t^{2}}+\left(\frac{\omega^{2}}{4}+\epsilon \cos \omega t\right) x=0
$$


and we seek an approximate solution valid for small $\epsilon$ by using the two-variable expansion perturbation method (Cole, 1968; Rand, 2004) (also known as multiple scales (Nayfeh and Mook, 1979)). This entails replacing time $t$ by two time variables, $\xi=t$ and $\eta=\epsilon t$, to give, if we neglect $O\left(\epsilon^{2}\right)$ terms,

$$
\frac{\partial^{2} x}{\partial \xi^{2}}+2 \epsilon \frac{\partial^{2} x}{\partial \xi \partial \eta}+\left(\frac{\omega^{2}}{4}+\epsilon \cos \omega \xi\right) x=0
$$

Next we expand $x=x_{0}+\epsilon x_{1}+\cdots$ and collect terms:

$$
\begin{aligned}
& \frac{\partial^{2} x_{0}}{\partial \xi^{2}}+\frac{\omega^{2}}{4} x_{0}=0 \Rightarrow x_{0}=A(\eta) \cos \frac{\omega \xi}{2}+B(\eta) \sin \frac{\omega \xi}{2} \\
& \frac{\partial^{2} x_{1}}{\partial \xi^{2}}+\frac{\omega^{2}}{4} x_{1}=-2 \frac{\partial^{2} x_{0}}{\partial \xi \partial \eta}-x_{0} \cos \omega \xi
\end{aligned}
$$

Substituting the expression for $x_{0}$ in (16) into (17) and eliminating secular terms, we obtain

$$
\frac{d A}{d \eta}=-\frac{B}{2 \omega}, \quad \frac{d B}{d \eta}=-\frac{A}{2 \omega},
$$

which gives

$$
\begin{aligned}
& A(\eta)=c_{3} \exp \left(\frac{\eta}{2 \omega}\right)+c_{4} \exp \left(-\frac{\eta}{2 \omega}\right) \\
& B(\eta)=-c_{3} \exp \left(\frac{\eta}{2 \omega}\right)+c_{4} \exp \left(-\frac{\eta}{2 \omega}\right)
\end{aligned}
$$

which furnishes us with the following approximate solution to equation (14):

$$
x=c_{3} \exp \left(\frac{\epsilon t}{2 \omega}\right)\left(\cos \frac{\omega t}{2}-\sin \frac{\omega t}{2}\right)+c_{4} \exp \left(-\frac{\epsilon t}{2 \omega}\right)\left(\cos \frac{\omega t}{2}+\sin \frac{\omega t}{2}\right) .
$$

Now by using equations (13) and (21) we can generate an analytical solution to equations (6)-(10). Starting with some given initial conditions, say $x(0)=1$ and $d x / d t(0)=0$, we obtain values for the arbitrary constants $c_{1}$ and $c_{2}$ in equation (13). Then by requiring continuity in $x$ and $d x / d t$ at $t=450$ we obtain values for the arbitrary constants $c_{3}$ and $c_{4}$ in equation (21). Similar calculations can be used for each of the regions (6)-(10). See Figure 11, which displays the resulting analytical solution. Comparison with Figure 5 shows that the simplified system has similar qualitative properties to the original system (4).

Next we use the foregoing analytical solution to investigate the effect of changing the initial condition phase $\gamma$ on the maximum values of $x$. The results are displayed in Figure 12, which is to be compared to Figure 6, which shows similar information obtained by numerically integrating the original system (1). Again the results show that the simplified system has similar qualitative properties to the original system (4). In particular, the curve marked B/A represents the amplification which occurs in the second tongue, and here, as in Figure 6, there is very little dependence on $\gamma$. 


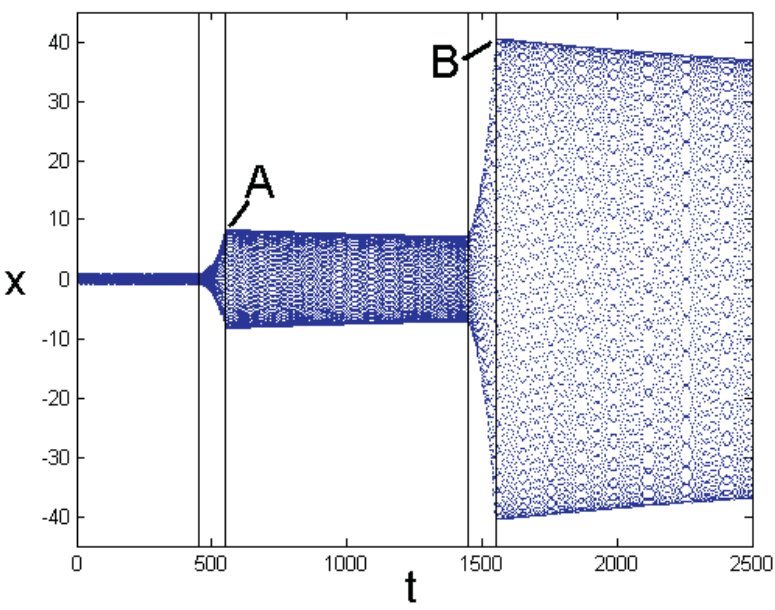

Figure 11. Analytical solution to simplified system (6)-(10) for the initial conditions $x(0)=1$ and $d x / d t(0)=0$. The vertical lines show nominal times at which the motion enters and exits the two resonant tongues (cf. Figure 5).

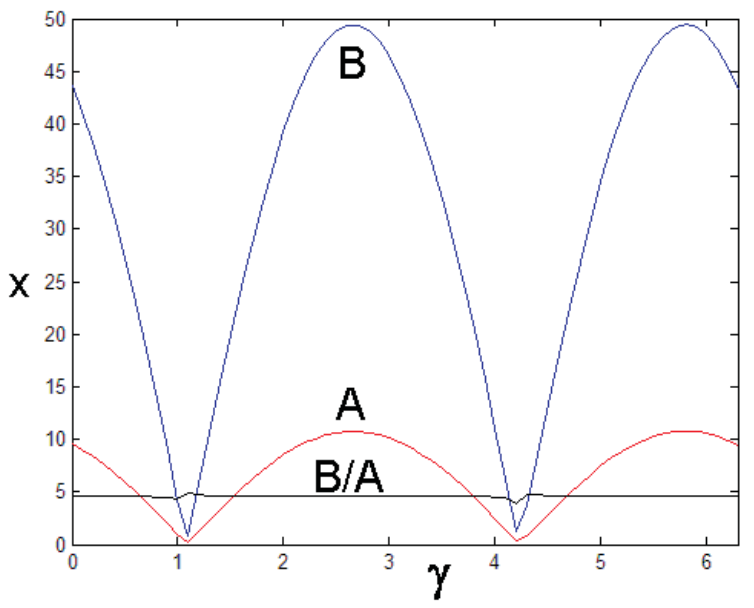

Figure 12. Maximum values of $x$ as a function of initial condition phase $\gamma$ for simplified system (6)-(10). The curves marked $A$ and $B$ refer to the local maximum values of $x$ associated respectively with the first and second resonant tongues, cf. Figure 11. The curve marked B/A shows the ratio of these maximum values and thus represents the amplification which occurs in the second tongue. Results obtained from equations (13),(21), being the analytical solution to equations (6)-(10). When comparing with Figure 6 , note the difference in scale of vertical axis. 
Now, however, because of the simplified nature of the system presented in Figures 11 and 12, we can understand why the amplification of the second tongue shows little response to a change in initial condition phase $\gamma$. The reason is that the solution (21) in the first tongue consists of both an exponentially growing term and an exponentially decaying term. Although both terms are typically present when the constants $c_{3}$ and $c_{4}$ are computed at $t=450$ (by requiring continuity in $x$ and $d x / d t$ ), the $c_{4}$ term will be greatly diminished at $t=550$ when the motion leaves the neighborhood of the first tongue. Neglecting the $c_{4}$ term, we obtain the following approximate expressions for $x$ and $d x / d t$ :

$$
\begin{aligned}
x & =c_{3} \exp \left(\frac{\epsilon t}{2 \omega}\right)\left(\cos \frac{\omega t}{2}-\sin \frac{\omega t}{2}\right), \\
\frac{d x}{d t} & =-\frac{\omega c_{3}}{2} \exp \left(\frac{\epsilon t}{2 \omega}\right)\left(\sin \frac{\omega t}{2}+\cos \frac{\omega t}{2}\right) .
\end{aligned}
$$

From these expressions we can see that the phase of the solution upon exiting the neighborhood of the first tongue, i.e. the ratio of $d x / d t$ to $x$, is independent of $c_{3}$ and $c_{4}$, and it is these constants which carry dependence on the original initial conditions. This explains why the amplification associated with second tongue generally shows no dependence on $\gamma$. However, if the original initial conditions are such that $c_{3}$ is zero, or is nearly zero, then the $c_{4}$ term cannot be neglected and the above argument is not applicable.

\section{EFFECT OF $\alpha_{i}$ ON THE SIMPLIFIED SYSTEM}

The effect of the initial conditions has been related to the effect of a temporary "unstable manifold" in each resonance tongue which generally causes the phase upon exit from the first resonant tongue to be independent of the initial conditions. We now consider the effect of varying the respective $\alpha_{i}$ on the amplification in each resonant tongue. In order to do so we need to generalize the simplified system (6)-(10) which referred to equation (4) to a corresponding system which refers to equation (5), i.e. which allows the $\alpha_{i}$ to be varied as parameters. Choosing the parameters $\sigma=0.5, \epsilon=0.1$ and $\mu=0.1$ as before, our simplified system becomes

$$
\begin{aligned}
x^{\prime \prime}+(0.5+0.001 t) x & =0 \text { for } 0<t<500-50 \alpha_{1}, \\
x^{\prime \prime}+\left(1+0.1 \alpha_{1} \cos 2 t\right) x & =0 \text { for } 500-50 \alpha_{1}<t<500+50 \alpha_{1}, \\
x^{\prime \prime}+(0.5+0.001 t) x & =0 \text { for } 500+50 \alpha_{1}<t<1500-50 \alpha_{2}, \\
x^{\prime \prime}+\left(2+0.1 \alpha_{2} \cos 2 \sqrt{2} t\right) x & =0 \text { for } 1500-50 \alpha_{2}<t<1500+50 \alpha_{2}, \\
x^{\prime \prime}+(0.5+0.001 t) x & =0 \text { for } 1500+50 \alpha_{2}<t .
\end{aligned}
$$




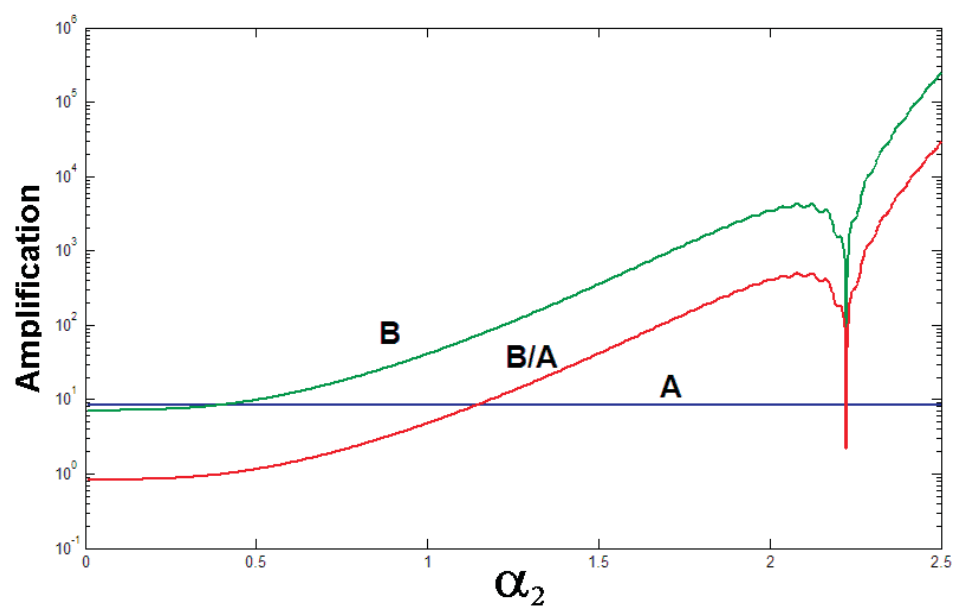

Figure 13. Maximum value of $x$ as a function of the coefficient of the parametric excitation in the second resonance tongue $\alpha_{2}$. The initial condition is chosen as $x(0)=1$ and $d x / d t(0)=0$. The curves marked $\mathrm{A}$ and $\mathrm{B}$ refer to the local maximum values of $x$ associated respectively with the first and second resonant tongues. The curve marked $\mathrm{B} / \mathrm{A}$ shows the ratio of these maximum values and thus represents the amplification which occurs in the second tongue. Results obtained from the analytic solution of the simplified system (24)-(28) with $\alpha_{1}=1$. When comparing with Figure 7 , note the difference in scale of vertical axis.

For brevity we omit the analytic solution of these equations, which are entirely analogous to that presented above for equations (6)-(10). Using this solution, the response of the simplified model was studied for two cases: (a) $\alpha_{1}$ was held fixed at 1 while $\alpha_{2}$ was allowed to vary from 0.1 to 2 ; (b) $\alpha_{2}$ was held fixed at 1 while $\alpha_{1}$ was allowed to vary from 0.1 to 2.5. This range, which is different from that used in the analysis of the original model, was chosen to show the similarity in the qualitative features of both systems.

The variation in the amplification of the simplified model (shown in Figures 13 and 14) shares several key features with the original equation. First, both show that for small values of $\alpha_{i}$, the amplification in the respective resonant tongue is approximately one. If $\alpha_{1}$ is small then the first resonance tongue has little effect on either the phase or the amplification because there is not enough time for the exponentially decaying term to die out (thus giving rise to an output phase which is largely invariant to initial conditions) and there is not enough time for the exponentially increasing term to grow, i.e. not enough time for significant amplification of the motion. Secondly, both Figures 13 and 14 show that as the respective $\alpha_{i}$ is varied, the growth is not monotonic, but rather exhibits local maxima and minima, indicating that the effect of the resonance tongue is not solely dependent on its width.

Analysis of the simplified model can explain the presence of the local extrema in the amplification curves. A plot of the exit-to-entry ratio of the vibration amplitude (based on the $\alpha_{i}$-dependent version of equation (21)) versus the phase at entry shows similar features to the curve $A$. The minimum amplification occurs when the $c_{3}$ coefficient goes to zero, i.e. when the entry phase to the first resonance tongue is $\phi_{i} \approx \arctan \left(\frac{\omega}{2} \tan \left(\frac{\pi}{4}-\frac{\omega t_{0}}{2}\right)\right)$ where 


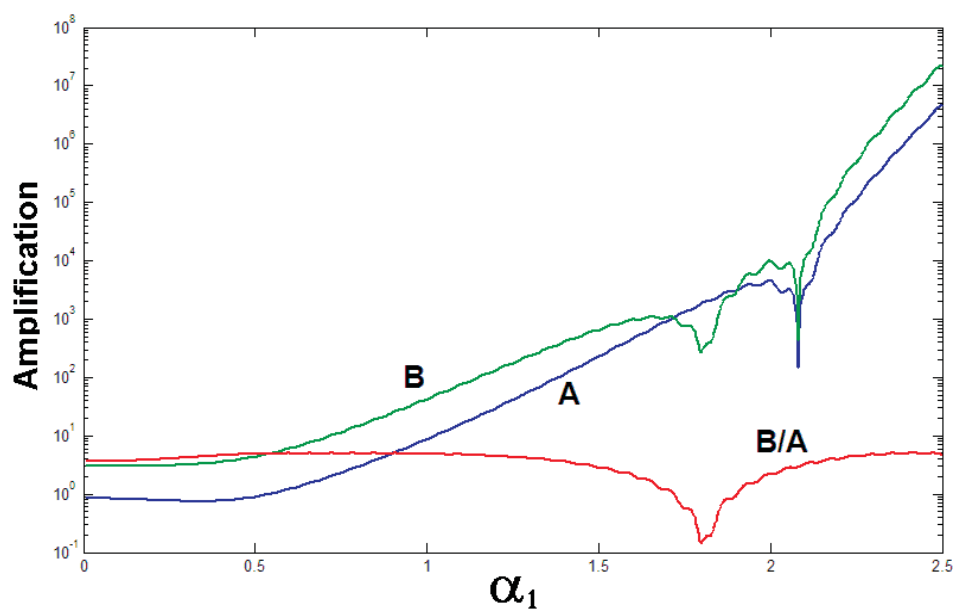

Figure 14. Maximum value of $x$ as a function of the coefficient of the parametric excitation in the first resonance tongue $\alpha_{1}$. The initial condition is chosen as $x(0)=1$ and $d x / d t(0)=0$. The curves marked $\mathrm{A}$ and $\mathrm{B}$ refer to the local maximum values of $x$ associated respectively with the first and second resonant tongues. The curve marked $\mathrm{B} / \mathrm{A}$ shows the ratio of these maximum values and thus represents the amplification which occurs in the second tongue. Results obtained from the analytic solution of the simplified system (24)-(28) with $\alpha_{2}=1$. When comparing with Figure 8 , note the difference in scale of vertical axis.

$t_{o}$ corresponds to the time that the motion enters the associated resonance tongue. Since the remaining term is exponentially decaying, the amplitude upon exit from the resonant tongue is less than the amplitude at entry, i.e. de-amplification occurs.

So how is this associated with the parametric excitation coefficients $\alpha_{i}$ ? Consider first the constant $\alpha_{1}$ case. The amplification in the second tongue will be minimum for a certain critical input phase. But the phase at entry to the second resonance tongue is the sum of the phase upon exiting the first resonance tongue (which does not depend on $\alpha_{2}$ in the simplified model) and the net change of phase accumulated during the nonresonant motion between exiting the first resonance tongue and entering the second resonance tongue. But the latter motion is primarily a rotation, the phase accumulation of which depends on the time between the exit from the first resonance tongue and the entry into the second resonance tongue, which, from equation (26) depends on $\alpha_{2}$.

A similar explanation can be used for the variation observed when $\alpha_{2}$ is held fixed and $\alpha_{1}$ is allowed to vary. The time when the motion hits the first nominal resonance tongue boundary will vary with $\alpha_{1}$; consequently the phase at entry to the first resonance tongue will vary. For the special value for which the entry phase coincides with the critical value, the amplification is minimized. Each critical entry phase value corresponds to the underlying temporary "stable manifold" in the resonance tongue. So we see that the variation of the amplification with initial condition phase $\gamma$ and the parametric excitation coefficients $\alpha_{i}$ are related to the same feature. 


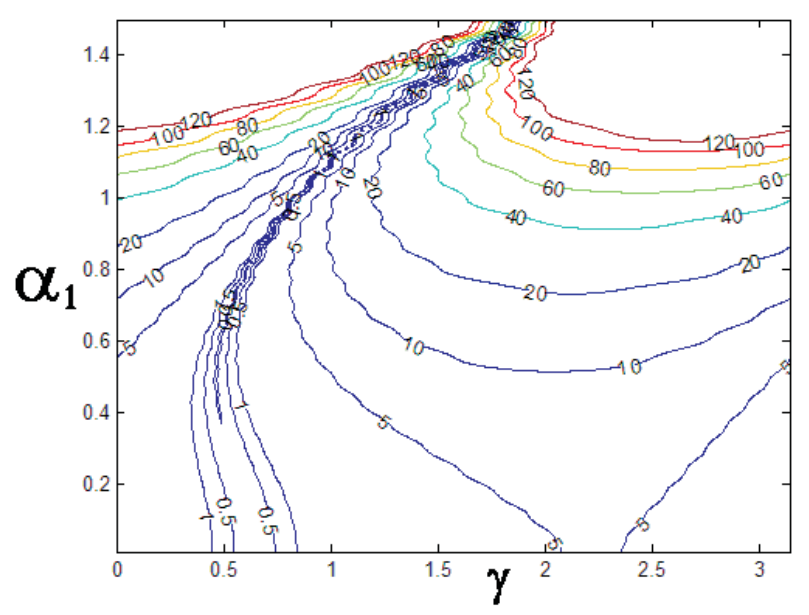

Figure 15. Contour plots of the maximum value of $x$ (i.e., point $\mathrm{B}$ in Figure 11) as a function of the coefficient of the parametric excitation in the first resonance tongue $\alpha_{1}$ and the initial condition parameter, $\gamma$. Results obtained from the analytic solution of the simplified system (24)-(28) with $\alpha_{2}=1$.

One major difference between the original equation (5) and the simplified system (24)(28) can be seen in the plots for constant $\alpha_{1}$. In the simplified model, Figure 13, the amplification in the first resonant tongue, curve $\mathrm{A}$, is a constant regardless of the value of $\alpha_{2}$. However, the numerical integration of equation (5), Figure 7, shows a variation in the amplification in the first resonant tongue, curve A. This indicates that, in the original equation, the parameter coefficient $\alpha_{2}$ of the second resonance tongue influences the amplification characteristics of the first resonance tongue. This should come as no surprise to the reader; there are no real resonances in equation (5). The dynamics of the system as it passes through the first "resonance tongue" is dependent on the coefficient of parametric excitation in the second tongue $\alpha_{2}$ and conversely, during passage through the second "resonance tongue", the response is influenced by the coefficient of parametric excitation in the first tongue $\alpha_{1}$.

To see the relationship between amplification and parameters $\alpha_{i}$ and initial condition phase $\gamma$, contour plots (Figures 15 and 16) were generated. Here again, there is a great similarity between the simplified model results and the results based on numerical integration of the original equation (5). The curve of minimum amplification in the $\gamma-\alpha_{i}$ plane does not coincide with the $\alpha_{i}=0$ line as it would if amplification depended only on the width of the resonance tongue. In Figure 16, the minimum amplification line is parallel to the $\gamma=0$. This means that for $\alpha_{1}$ fixed and $\alpha_{2}$ varying, the maximum amplification takes place for the same initial condition. This is because the motion becomes greatly de-amplified in the first resonance tongue since it is caught on the "stable manifold". The fact that the minimum amplification curve is parallel to the $\alpha_{2}$ axis when $\alpha_{1}$ is fixed indicates that the simplified model behaves as if $\alpha_{2}$ has no effect on the dynamics of the first resonance tongue, a property which, as discussed above, is not true for the original equation (5), as can be seen from its analogous contour plot (Figure 10). 


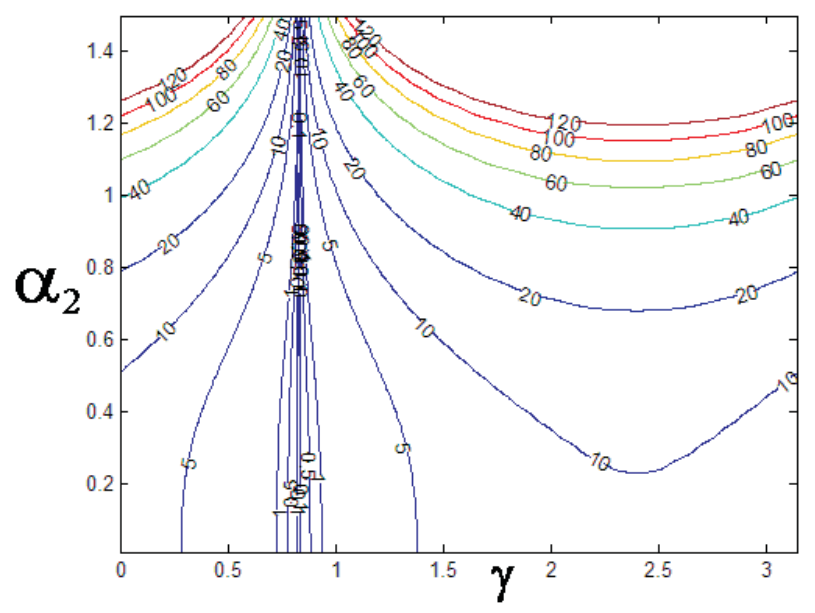

Figure 16. Contour plots of the maximum value of $x$ (i.e., point $\mathrm{B}$ in Figure 11) as a function of the coefficient of the parametric excitation in the second resonance tongue $\alpha_{2}$ and the initial condition parameter, $\gamma$. Results obtained from the analytic solution of the simplified system (24)-(28) with $\alpha_{1}=1$.

\section{THREE TONGUES}

We extend our foregoing discussion of slow passage through two-tongue systems by considering the following three-tongue system:

$$
\frac{d^{2} x}{d t^{2}}+(\delta+\epsilon(\cos 2 t+\cos 2 \sqrt{2} t+\cos 2 \sqrt{3} t)) x=0 \quad \text { where } \quad \delta=\sigma+\epsilon^{2} \mu t
$$

Here the forcing frequencies have been chosen so that the tongues are centered respectively at $\delta=1,2$ and 3. Figure 17 shows the results of numerical integration of equation (29) in which $\sigma=0.5, \epsilon=0.1$ and $\mu=0.1$ for the initial conditions $x(0)=1$ and $d x / d t(0)=0$. Figures 18 and 19 show the maximum values of $x$ as a function of the initial condition phase $\gamma$. Examination of these Figures shows that, as one might expect, in general each additional resonance tongue produces additional growth. However, as a study of Figure 18 shows, the total amplification can be controlled and even minimized to the point of de-amplification by choosing the initial-condition phase so that the phase upon entering the first tongue is appropriately selected. From Figure 19 we see that in general the amplification in the second and third tongues is relatively unaffected by the initial condition phase due to the saddle-like nature of the flow inside the first tongue, as discussed above. An exception occurs when the spacing between the tongues is such that the motion happens to enter one of the tongues along its "stable manifold". 


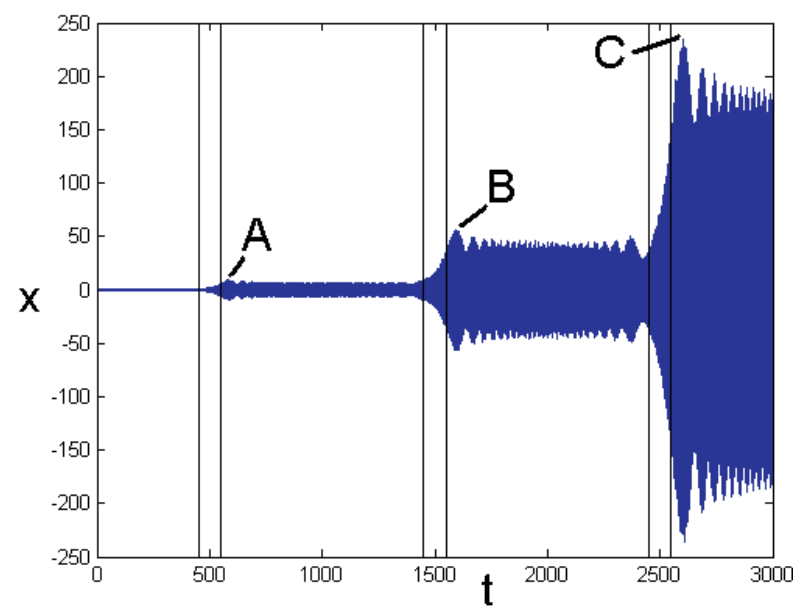

Figure 17. Numerical integration of equation (29) for $\sigma=0.5, \epsilon=0.1, \mu=0.1$ and for the initial conditions $x(0)=1$ and $d x / d t(0)=0$. The vertical lines show nominal times at which the motion enters and exits the three resonant tongues. The letters $A, B$ and $C$ refer to the local maximum values of $x$ associated respectively with the first, second and third resonant tongues, cf. Figure 6.

\section{CONCLUSION}

Through numerical integration we found that the two-tongue Mathieu equation can exhibit a wide range of amplification and de-amplification for varying $\alpha_{i}$ parameter values and initial conditions. Using a simplified model of the system, we showed that this feature is dependent on a temporary stable-unstable manifold structure which causes almost all motions to exit the first resonance tongue at a fixed phase for given parameters $\alpha_{i}, \sigma, \mu$ and $\epsilon$. (This assumes that the first tongue is sufficiently wide, i.e. that $\alpha_{1}$ is sufficiently large.)

In situations where it is desirable to minimize the growth of the solution after a slow passage through a multi-tongue system, de-amplification may be induced by choosing the initial conditions and the $\alpha_{i}$ parameters appropriately. In the case of a system which has only a single tongue, it is possible to minimize amplification by choosing the initial condition phase $\gamma$ appropriately. In systems with two or more tongues, adjusting the initial condition phase will not in general permit de-amplification to occur in any but the first tongue. However, in such a case we may adjust the relative forcing amplitudes $\alpha_{i}(i=2,3, \ldots)$, which will effectively control the phase of the solution at the entrance to the $i^{t h}$ tongue, and which may therefore be used to minimize the amplitude of the final response.

As an example of this process, we consider the three-tongue system:

$$
\frac{d^{2} x}{d t^{2}}+\left(\delta+\epsilon\left(\cos 2 t+\alpha_{2} \cos 2 \sqrt{2} t+\alpha_{3} \cos 2 \sqrt{3} t\right)\right) x=0 \quad \text { where } \quad \delta=\sigma+\epsilon^{2} \mu t
$$

in which $\sigma=0.5, \epsilon=0.1$ and $\mu=0.1$. Equation (30) is a generalization of the previously considered three-tongue system (29) in which the coefficients $\alpha_{2}$ and $\alpha_{3}$ of (30) were taken as unity in (29). Based on Figure 19, we choose the initial condition phase $\gamma \approx 0.37$, giving 


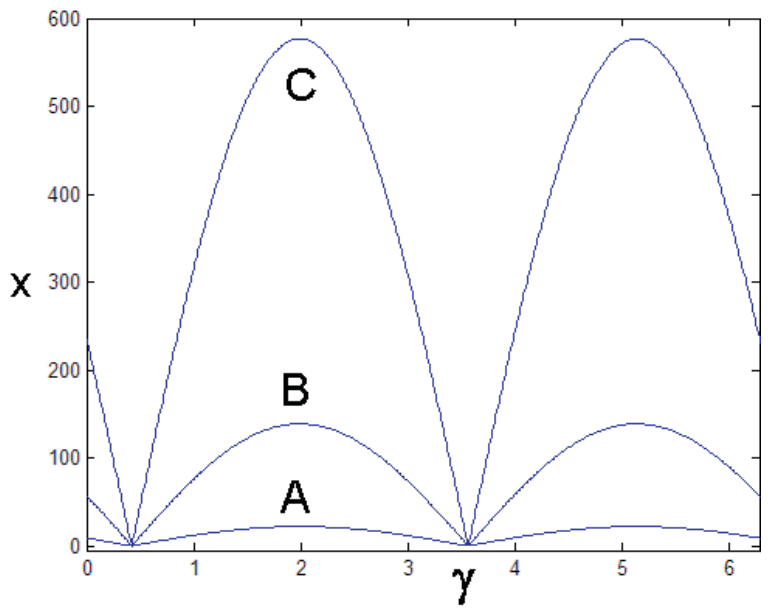

Figure 18. Maximum values of $x$ as a function of initial condition phase $\gamma$ for equation (29) with $\sigma=0.5$, $\epsilon=0.1$ and $\mu=0.1$. The curves marked $\mathrm{A}, \mathrm{B}$ and $\mathrm{C}$ refer to the local maximum values of $x$ associated respectively with the first, second and third resonant tongues, cf. Figure 17. Results obtained by numerically integrating equation (29).

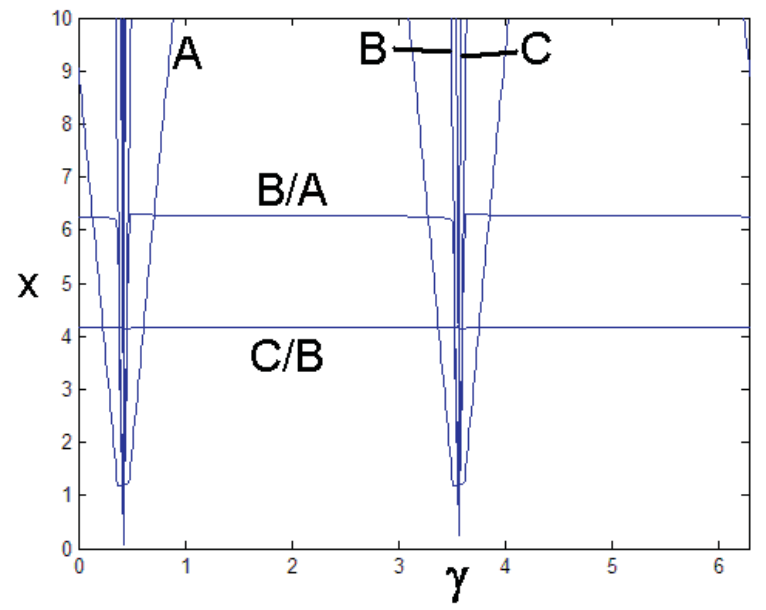

Figure 19. This Figure is an enlargement of the lower portion of Figure 18, and includes two additional curves. The curve marked $B / A$ shows the ratio of maximum values $B$ and $A$, and thus represents the amplification which occurs in the second tongue. The curve marked $C / B$ shows the ratio of maximum values $C$ and $B$, and thus represents the amplification which occurs in the third tongue. 


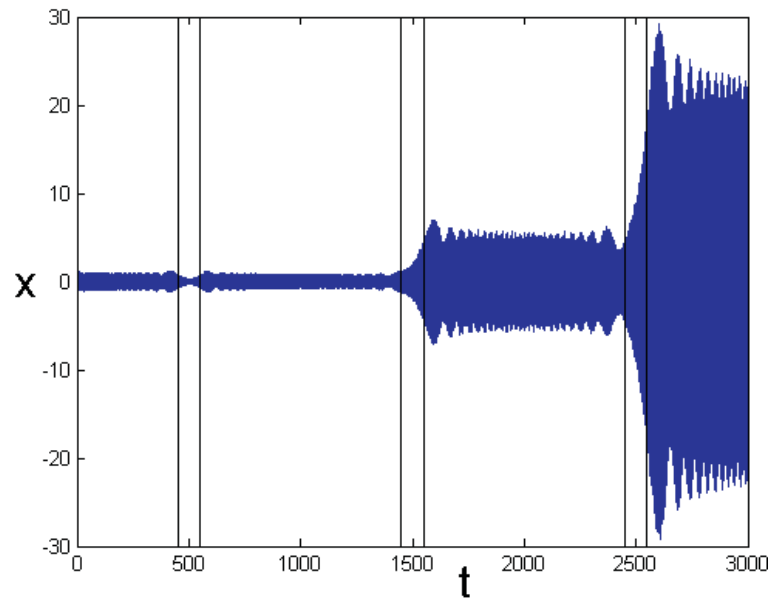

Figure 20. Numerical integration of equation (30) for $\sigma=0.5, \epsilon=0.1, \mu=0.1, \alpha_{2}=1, \alpha_{3}=1$ and for the initial conditions $x(0)=\cos \gamma=0.93233, d x / d t(0)=\sin \gamma=0.36162$. The vertical lines show nominal times at which the motion enters and exits the three resonant tongues. By selecting the initial-condition phase $\gamma=0.37$, de-amplification has been achieved in the first tongue, but not in the second or third tongues. Note the difference in vertical scale compared to Figure 17.

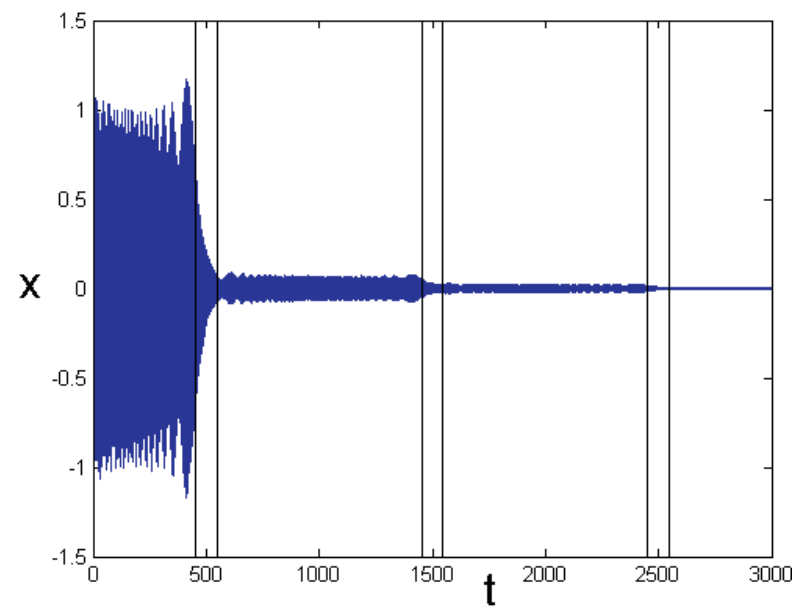

Figure 21. Numerical integration of equation (30) for $\sigma=0.5, \epsilon=0.1, \mu=0.1, \alpha_{2}=0.88, \alpha_{3}=0.9875$ and for the initial conditions $x(0)=\cos \gamma=0.93233, d x / d t(0)=\sin \gamma=0.36162$. The vertical lines show nominal times at which the motion enters and exits the three resonant tongues. By selecting both initial condition phase $\gamma=0.37$ and the parameter $\alpha_{2}=0.88$ and $\alpha_{3}=0.9875$, de-amplification has been achieved in all three tongues. Note the difference in vertical scale compared with Figure 20. 
the initial conditions $x(0)=\cos \gamma=0.93233, d x / d t(0)=\sin \gamma=0.36162$. This choice achieves de-amplification in the first tongue, but not in the second or third tongues. See Figure 20.

In order to achieve de-amplification in all three tongues, we again choose the initial condition phase $\gamma$ as 0.37 , and in addition we choose the parameters $\alpha_{2}=0.88$ and $\alpha_{3}=$ 0.9875 , values obtained by numerical experimentation. The result is shown in Figure 21.

Acknowledgments. Support received from the Moroccan American Commission for Educational and Cultural Exchange through the Fulbright Program is acknowledged by author SMS. The hospitality of the Department of Theoretical and Applied Mechanics, Cornell University, is gratefully acknowledged.

\section{REFERENCES}

Bender, C. M. and Orszag, S. A., 1978, Advanced Mathematical Methods for Scientists and Engineers, McGraw-Hill, New York.

Cole, J. D., 1968, Perturbation Methods in Applied Mathematics, Blaisdell, Waltham, MA.

Nayfeh, A. H. and Mook, D. T., 1979, Nonlinear Oscillations, Wiley, New York.

Ng, L., Rand, R., and O’Neil, M., 2003, “Slow passage through resonance in Mathieu's equation,” Journal of Vibration and Control 9, 685-707.

Rand, R. H., 2004, Lecture Notes on Nonlinear Vibrations, published on-line by The Internet-First University Press, http://dspace.library.cornell.edu/handle/1813/79.

Zounes, R. S. and Rand, R.H., 1998, “Transition Curves in the Quasiperiodic Mathieu Equation,” SIAM Journal of Applied Mathematics 58, 1094-1115. 